\title{
Quantum Rabi model in the Brillouin zone with ultracold atoms
}

\author{
Simone Felicetti, ${ }^{1}$ Enrique Rico, ${ }^{2,3}$ Carlos Sabin, ${ }^{4}$ Till Ockenfels, ${ }^{5}$ Johannes Koch, ${ }^{5}$ Martin Leder, ${ }^{5}$ Christopher Grossert,${ }^{5}$ \\ Martin Weitz, ${ }^{5}$ and Enrique Solano ${ }^{2,3}$ \\ ${ }^{1}$ Laboratoire Matériaux et Phénomènes Quantiques, Sorbonne Paris Cité, Université Paris Diderot, CNRS UMR 7162, 75013 Paris, France \\ ${ }^{2}$ Department of Physical Chemistry, University of the Basque Country UPV/EHU, Apartado 644, E-48080 Bilbao, Spain \\ ${ }^{3}$ IKERBASQUE, Basque Foundation for Science, Maria Diaz de Haro 3, E-48013 Bilbao, Spain \\ ${ }^{4}$ Instituto de Física Fundamental, CSIC, Serrano 113-bis, E-28006 Madrid, Spain \\ ${ }^{5}$ Institut für Angewandte Physik der Universität Bonn, Wegelerstrasse 8, D-53115 Bonn, Germany
}

(Received 7 July 2016; published 18 January 2017)

\begin{abstract}
The quantum Rabi model describes the interaction between a two-level quantum system and a single bosonic mode. We propose a method to perform a quantum simulation of the quantum Rabi model, introducing an implementation of the two-level system provided by the occupation of Bloch bands in the first Brillouin zone by ultracold atoms in tailored optical lattices. The effective qubit interacts with a quantum harmonic oscillator implemented in an optical dipole trap. Our realistic proposal allows one to experimentally investigate the quantum Rabi model for extreme parameter regimes, which are not achievable with natural light-matter interactions. When the simulated wave function exceeds the validity region of the simulation, we identify a generalized version of the quantum Rabi model in a periodic phase space.
\end{abstract}

DOI: 10.1103/PhysRevA.95.013827

\section{INTRODUCTION}

The Rabi model [1] is a semiclassical description of the dipolar interaction of a nuclear spin with electromagnetic radiation. Its full quantum version, known as the quantum Rabi model, has been applied more generally to describe the interaction between a two-level quantum system and a single bosonic mode, regardless of their specific physical origin. In the strong-coupling regime, where the coupling strength is larger than dissipation rates but small compared to the system characteristic frequencies, the quantum Rabi model can be reduced via a rotating-wave approximation to the JaynesCummings model [2]. The latter has been used for decades to explain a plethora of experiments [3-5] in quantum optics and condensed matter, such as cavity quantum electrodynamics (QED), trapped ions, circuit QED, and quantum dots.

More recently, it was experimentally demonstrated that the ultrastrong-coupling regime can also be achieved [6-11], where the coupling strength is large enough to break the rotating-wave approximation and the full quantum Rabi model must be considered. The interest in the ultrastrong-coupling regime is motivated by novel fundamental features [12-17] and potential computational benefits [18-21]. Despite its ubiquity, analytical solutions for the quantum Rabi model spectrum were developed only recently [22], prompting further theoretical efforts to study generalizations of the quantum Rabi model, including anisotropic couplings [23], two-photon interactions [24-26], and the Dicke model [27]. In addition, significant efforts have been devoted to reproducing these models using different quantum technologies [28-32].

Ultracold atoms represent one of the most advanced quantum platforms for the implementation of analog quantum simulations [33]. They have mostly been associated with the implementation of quantum many-body and condensed-matter models. Spinlike degrees of freedom have been implemented with ultracold atoms using internal electronic transitions [34-36]. Alternatively, the creation of two-level systems with atomic quantum dots [37,38] or double-well potentials [39] has been proposed. Remarkably, relativistic effects [40-42] have also been simulated using ultracold atoms.

Here, we propose a quantum simulation of the quantum Rabi model with cold atoms in an optical lattice. The effective two-level quantum system is simulated by different Bloch bands in the first Brillouin zone, and the bosonic mode is represented by the motion of the atomic cloud in a superimposed harmonic optical-trap potential. The qubit energy spacing is proportional to the periodic lattice depth, while the interacting bosonic mode is intrinsic in the qubit definition. When the edge of the Brillouin zone is reached, we find that a generalized version of the quantum Rabi model in periodic phase space is realized.

We show that our method, feasible with current technology, can access extreme parameter regimes of the quantum Rabi model. This will allow for the experimental study of the transition between the deep strong-coupling (DSC) regime [43], where the coupling strength is larger than bosonic-mode frequency, and the dispersive deep strong-coupling (dDSC) regime, where in addition the frequency of the qubit is much larger than the frequency of the bosonic mode. The complexity of such a model has been recently highlighted by the prediction of a phase transition, even in the single-qubit case, when the ratio between the qubit and the bosonic frequency tends to infinity [44].

This paper is organized as follows. In Sec. II, we introduce the model and we discuss the analogy with the quantum Rabi model (QRM). In Sec. III, we provide details on the proposed implementation, discussing the parameter regimes that can be accessed with current technology and the measurement techniques. In Sec. IV, we report the results of numerical simulations that show examples of interesting dynamics that could be reproduced. Finally, in Sec. V we summarize our results.

\section{THE MODEL}

The system considered here is composed of a cloud of ultracold atoms exposed to two laser-induced potentials: a 
periodic lattice and a harmonic trap. When the atom density is sufficiently low, interactions among the atoms are negligible, and the system can be described with a single-particle Hamiltonian, composed of the periodic term $H_{\mathrm{P}}$ and a quadratic term,

$$
\hat{H}=\hat{H}_{\mathrm{P}}+\frac{m \omega_{0}^{2}}{2} \hat{x}^{2}, \quad \hat{H}_{\mathrm{P}}=\frac{\hat{p}^{2}}{2 m}+\frac{V}{2} \cos \left(4 k_{0} \hat{x}\right),
$$

where $\hat{p}=-i \hbar \frac{\partial}{\partial x}$ and $\hat{x}$ are the momentum and position of an atom of mass $m$, respectively. Here, $\omega_{0}$ is the angular frequency of the atom motion in the harmonic trap, while $V$ and $4 k_{0}$ are the depth and wave vector of the periodic potential, respectively. The periodic lattice results from a four-photon interaction with a driving field $[41,45,46]$ of wave vector $k_{0}$.

In the following, we will assume that the harmonic trap is slowly varying on the length scale of the periodic potential. Under this assumption, the most suitable basis is given by the Bloch functions $\left\langle x \mid \phi_{n_{b}}(q)\right\rangle=\phi_{n_{b}}(q, x)=e^{i q x / \hbar} u_{n_{b}}(x)$, with $q$ the quasimomentum and $n_{b}$ the band index, while $u_{n_{b}}(x)$ must be a periodic function with the same periodicity of the periodic potential. Without loss of generality, we define $u_{n_{b}}(x)=$ $e^{-i 2 k_{0} x} e^{i 4 n_{b} k_{0} x}$, where we have added the phase $e^{-i 2 k_{0} x}$ to the $u_{n_{b}}(x)$ functions definition in order to obtain a convenient first Brillouin zone, $q \in\left(-2 \hbar k_{0}, 2 \hbar k_{0}\right]$. Notice that the Bloch functions are identified by a discrete quantum number, the band index $n_{b}$, and a continuous variable, the quasimomentum $q$. Hence, we can define continuous and discrete degrees of freedom, and rewrite the Bloch basis as $\left|\phi_{n_{b}}(q)\right\rangle=|q\rangle\left|n_{b}\right\rangle$.

First, let us consider the periodic part $\hat{H}_{\mathrm{P}}$ of the system Hamiltonian; later we will discuss the effect of the harmonic trap. It is straightforward to see that the momentum operator is diagonal in the Bloch basis, while the periodic potential introduces a coupling between adjacent bands,

$$
\begin{aligned}
\hat{H}_{\mathrm{P}}|q\rangle\left|n_{b}\right\rangle= & \frac{1}{2 m}\left[q+\left(2 n_{b}-1\right) 2 \hbar k_{0}\right]^{2}|q\rangle\left|n_{b}\right\rangle \\
& +\frac{V}{4}\left(|q\rangle\left|n_{b}+1\right\rangle+|q\rangle\left|n_{b}-1\right\rangle\right) .
\end{aligned}
$$

Let us now include the quadratic term of Eq. (1) in our treatment. In the Bloch basis, we can write

$$
\left\langle\tilde{q}, \tilde{n}_{b}\left|\hat{x}^{2}\right| q, n_{b}\right\rangle=\int_{-\infty}^{+\infty} d x x^{2} e^{i\left[4\left(n_{b}-\tilde{n}_{b}\right) k_{0}+(q-\tilde{q}) / \hbar\right] x} .
$$

Considering diagonal elements in the band index, i.e., setting $\tilde{n}_{b}=n_{b}$, we have $\left\langle\tilde{q}, n_{b}\left|\hat{x}^{2}\right| q, n_{b}\right\rangle=-\hbar^{2}\left\langle\tilde{q}, n_{b}\left|\frac{\partial^{2}}{\partial q^{2}}\right| q, n_{b}\right\rangle$. Hence, we see that the harmonic potential introduces an operator, diagonal in the qubit Hilbert space, which can be expressed as $\hat{x}=-i \hbar \frac{\partial}{\partial q}$, in the Bloch basis. This allows us to define the quasimomentum operator $\hat{q}$ and the position operator $\hat{x}$, which satisfy the commutation relation $[\hat{x}, \hat{q}]=i \hbar$.

On the other hand, for $\tilde{n}_{b} \neq n_{b}$, the integral in Eq. (3) is maximized if the relation $4 \hbar k_{0}\left(n_{b}-\tilde{n}_{b}\right)=\tilde{q}-q$ is satisfied. Hence, the quadratic potential introduces a coupling between neighboring bands, for states whose momenta satisfy $\tilde{q}-q=4 \hbar k_{0}$, of the kind $\left(\left|2 \hbar k_{0}, n_{b}\right\rangle\left\langle-2 \hbar k_{0}, n_{b}+1\right|+\right.$ H.c. $)$. This effective coupling is due to the periodicity of the quasimomentum, which mixes the bands at the boundaries of the Brillouin zone. Such a coupling can be neglected as far as the system dynamics involves only values of the

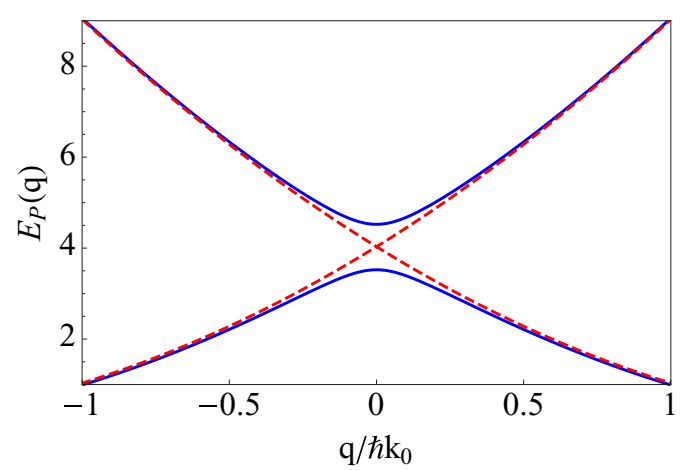

FIG. 1. Band structure for an optical lattice potential. Comparison of the dispersion relation for the first and second bands for a latticepotential depth of $V=2 E_{r}$ (solid blue line) and a free particle (dashed red line). The gap between the first and second bands corresponds to the effective qubit energy splitting.

quasimomentum $\hat{q}$ included within the first Brillouin zone. This condition marks the limits of validity of the identification of the quasimomentum as a harmonic-oscillator variable. When the exponent in Eq. (3) is not vanishing, the argument of the integral is fast oscillating and the corresponding matrix elements are negligible.

\section{A. Equivalence with the quantum Rabi model}

We assume now that the system dynamics is restricted to the two bands with lowest energy $\left(n_{b}=0,1\right)$, as shown in Fig. 1. The validity of this assumption is granted by energy conservation, when the system is initialized in this subspace. Accordingly, we can truncate Eq. (2) and rewrite in the Bloch basis the periodic part $\hat{H}_{\mathrm{P}}$ of the Hamiltonian as

$$
\hat{H}_{\mathrm{P}}(q)=\frac{1}{2 m}\left(\begin{array}{cc}
q^{2}+4 \hbar k_{0} q & 0 \\
0 & q^{2}-4 \hbar k_{0} q
\end{array}\right)+\frac{V}{4}\left(\begin{array}{ll}
0 & 1 \\
1 & 0
\end{array}\right) .
$$

Similarly, we can rewrite Eq. (3), keeping only the nonvanishing terms of the quadratic trapping potential that involve the two lowest bands as

$$
\hat{x}^{2}=-\hbar^{2} \frac{\partial^{2}}{\partial q^{2}}\left(\begin{array}{ll}
1 & 0 \\
0 & 1
\end{array}\right)+\Omega\left(\left|2 \hbar k_{0}, 0\right\rangle\left\langle-2 \hbar k_{0}, 1\right|+\text { H.c. }\right)
$$

where $\Omega=\left\langle-2 \hbar k_{0}, 1\left|\hat{x}^{2}\right| 2 \hbar k_{0}, 0\right\rangle$. We define the annihilation operator $\hat{a}=\sqrt{\frac{m \omega_{0}}{2 \hbar}}\left(-i \hbar \frac{\partial}{\partial q}+\frac{i}{m \omega_{0}} \hat{q}\right)$ and creation operator $\hat{a}^{\dagger}$, respectively. In the following, we assume that the system state is fully contained in the first Brillouin zone, i.e., that its overlap with the quasimomentum states $q= \pm 2 \hbar k_{0}$ vanishes. Accordingly, the umklapp term proportional to $\Omega$ in Eq. (5) can be neglected. We will discuss the implication of this assumption at the end of this section. Finally, the total system Hamiltonian, up to the umklapp term, can be rewritten as

$$
\hat{H}=\hbar \omega_{0} \hat{a}^{\dagger} \hat{a}+\frac{\hbar \omega_{q}}{2} \sigma_{z}+i \hbar g \sigma_{x}\left(a^{\dagger}-a\right),
$$

which corresponds to the quantum Rabi Hamiltonian where we have defined the effective qubit energy spacing $\omega_{q}=\frac{V}{2 \hbar}$ and the interaction strength $g=2 k_{0} \sqrt{\frac{\hbar \omega_{0}}{2 m}}$. In order to obtain the standard form of the quantum Rabi model, we have rotated 
the qubit basis with the unitary operator $U=\frac{1}{\sqrt{2}}\left(\begin{array}{rr}1 & -1 \\ 1 & 1\end{array}\right)$. The Pauli matrices are defined in the rotated basis and, using the notation for the Bloch bands, they can be written as

$$
\begin{aligned}
& \sigma_{x}=\left|n_{b}=0\right\rangle\left\langle n_{b}=0|-| n_{b}=1\right\rangle\left\langle n_{b}=1\right|, \\
& \sigma_{z}=\left|n_{b}=1\right\rangle\left\langle n_{b}=0|+| n_{b}=0\right\rangle\left\langle n_{b}=1\right| .
\end{aligned}
$$

Notice that in the standard form of the quantum Rabi model, the qubit-field coupling is usually written in terms of the position operator, while in Eq. (6) it appears in terms of the momentum operator. The two definitions are equivalent up to a global phase factor.

The full system Hamiltonian of Eq. (1) resembles the quantum Rabi model only when the effective coupling between different bands induced by the harmonic potential can be neglected. Such an approximation holds as long as the system wave function $\langle q \mid \psi(t)\rangle$ is completely included in the first Brillouin zone. Clearly, this constraint limits the proposed implementation to values of the momentum $\hat{q}$ smaller in modulus than $2 \hbar k_{0}$. In the following, we will show that this constraint does not impede to observe the highly nontrivial behavior of the quantum Rabi model in the DSC and dDSC regimes. The DSC regime was introduced theoretically in [43] and it is generically characterized by $g>\omega_{0}$. However, in the $\mathrm{dDSC}$ regime, we have the condition $\omega_{q} \gg \omega_{0}$. Interestingly, some key features of the DSC regime are reproduced even when the periodicity of the quasimomentum becomes relevant for the system dynamics.

\section{B. Parameters regimes of the quantum Rabi model}

The spectrum and the dynamical evolution of the quantum Rabi model have very different features depending on the ratio between the interaction strength $g$ and the qubit and bosonic mode energies $\omega_{q}$ and $\omega_{0}$. Three different parameter regimes can be identified, in which the model behaves in a qualitatively different way.

In the strong-coupling (SC) regime, the interaction strength $g$ is large with respect to decay channels but small compared to the system characteristic frequencies $g / \omega_{0} \ll 1$ and $g / \omega_{q} \ll$ 1. In this case, a rotating-wave approximation (RWA) can be applied, and the interaction term in Eq. (6) can be rewritten as $i \hbar g \sigma_{x}\left(a^{\dagger}-a\right) \rightarrow i \hbar g\left(\sigma_{-} a^{\dagger}-\sigma_{+} a\right)$. We defined the qubit raising and lowering qubit operators $\sigma_{ \pm}=\left(\sigma_{x} \pm i \sigma_{y}\right) / 2$. Under RWA, the total number of excitation $\hat{N}=a^{\dagger} a+\sigma_{z}$ is conserved. The resulting Hamiltonian, dubbed the JaynesCummings model [2], is analytically solvable and it has proven useful to describe a plethora of experiments in the quantum regime.

As the coupling strength becomes larger, the rotating-wave approximation is not applicable and the system enters the ultrastrong-coupling (USC) regime, defined by the condition $0.1 \lesssim g / \omega_{0} \lesssim 1$, in the resonant or near-resonant case $\omega \approx \omega_{q}$. The continuous symmetry $\hat{N}$ breaks down into a discrete $\mathbb{N}_{2}$ symmetry, $\hat{\Pi}=-\sigma_{z} e^{i \pi a^{\dagger} a}$, representing the parity of the total excitation number. In this regime, the quantum Rabi Hamiltonian is not analytically solvable, even if approximated solutions can be found for limiting cases [47]. An analytical solution for the eigenspectrum has also been derived [22], but it is not in a closed form and it does not entail computational benefits.

The system enters the deep strong-coupling regime [43] when both the ratio $g / \omega_{0}$ and $g / \omega_{q}$ are significant higher than 1. Under this condition, the system time evolution is well described by the slow-qubit approximation, which consists in neglecting the energy splitting of the qubit, setting $\omega_{q}=0$. In our case, the Hamiltonian of Eq. (6) will be reshaped into $\hat{H}_{\mathrm{DSC}}=\omega_{0} a^{\dagger} a-i g \sigma_{x}\left(a^{\dagger}-a\right)$. This approximation is intuitively motivated by the rapid growth of the number of photons, which makes the higher states of the Fock basis the most relevant in the system dynamics. Given that the norms of the creation and annihilation operators are proportional to the Fock number, while the Hamiltonian term $\frac{\omega_{q}}{2} \sigma_{z}$ remains constant, the latter becomes negligible for high photon number states. Under this approximation, the Hamiltonian can be diagonalized using the transformation

$$
D^{\dagger}(\alpha) H_{\mathrm{DSC}} D(\alpha)=\omega_{0} a^{\dagger} a+\frac{g^{2}}{\omega_{0}^{2}}-\frac{2 g^{2}}{\omega_{0}},
$$

where we defined $\alpha=-i \frac{g}{\omega_{0}} \sigma_{x}$ and the operator $D(\alpha)=$ $\exp \left\{\alpha a^{\dagger}-\alpha^{*} a\right\}$, which corresponds to a conditional displacement dependent on the qubit state.

On the other hand, when the qubit energy splitting is comparable to the coupling strength $\omega_{q} \sim g$, the slow-qubit approximation cannot be applied and the diagonalization of Eq. (8) cannot be applied. We will call dispersive DSC (dDSC) the regime where the coupling strength $g$ is large with respect to the bosonic-mode frequency $\omega_{0}$, and it is comparable or smaller than the qubit energy splitting $\omega_{q}$. A low-energy effective Hamiltonian has been found in the limit $\omega_{q} / \omega_{0} \rightarrow \infty$, where the system exhibits a quantum phase transition [44]. The present proposal allows reaching unprecedented values of the ratio $\omega_{q} / \omega_{0}$, so it can in principle approach the limit in which the phase transition takes place. Notice that there are no available analytical or approximated solutions to describe a large interval of parameters at the crossover between the DSC and the dDSC regime.

\section{IMPLEMENTATION}

Before going into the details of the phenomena that can be observed, let us discuss the feasibility of the present proposal. In this section, we report which parameter regimes can be accessed with current technology, and we briefly describe state preparation and measurement techniques. In particular, we consider previous experiments of ultracold rubidium atoms in optical lattices, where Fourier-synthesized lattice potentials are used in order to tailor the atomic dispersion relation $[42,48]$.

\section{A. Accessibility of parameter regimes}

The parameter regimes that can be accessed are established by the relative weights of the parameters $\omega_{0}, \omega_{q}=\frac{V}{2 \hbar}$ and $g=2 k_{0} \sqrt{\frac{\hbar \omega_{0}}{2 m}}$, defined in Eq. (6). We will consider as fixed parameters the wavelength of the lattice laser $\lambda=783.5 \times$ $10^{-9} \mathrm{~m}$ for technical reasons and, obviously, the rubidium atomic mass $m=1.44 \times 10^{-25} \mathrm{~kg}$. The wave vector is given 
by $k_{0}=\frac{2 \pi}{\lambda}$. On the other hand, the periodic lattice can be tuned over a wide intensity range, from $V=0$ (absence of lattice) to about $V / \hbar=2 \pi \times 18 \mathrm{kHz}$, corresponding to $V=4.8 E_{r}$, where we defined the recoil energy $E_{r}=\frac{\hbar^{2} k_{0}^{2}}{2 m}$. The frequency of the harmonic trap can also be tuned from $\omega_{0}=2 \pi \times 0.5$ to $\omega_{0}=2 \pi \mathrm{kHz}$.

First, notice that the value of the effective coupling strength is intrinsically linked to the trap frequency, $g \sim \sqrt{\omega_{0}}$, and hence the ratio $g / \omega_{0}$ is tunable only over a narrow range of extremely high values, from $g / \omega_{0} \approx 7 \omega_{0}$ to $g / \omega_{0} \approx 11$. However, the tunability of the ratio between the coupling strength and the effective qubit energy spacing allows us to explore a large region of parameters at the transition between the DSC and the dDSC regimes. Indeed, the value of $\omega_{q}$ can be made large enough to make the qubit-free Hamiltonian dominant, $\omega_{q} / \omega_{0} \approx 30$, or small enough to make its energy contribution negligible.

\section{B. State preparation and measurement}

A trapping potential for atoms can be realized by superimposing a dipole potential generated by a focused far-reddetuned laser beam. To minimize the interatomic interactions, it will be desirable to operate with a moderate number of atoms, typically a few thousand. After initializing, the momentum of the atoms must be manipulated in order to produce relevant states of the simulated qubit and bosonic mode, according to the encoding defined in Sec. II A.

Notice that the qubit state is encoded in the occupation of the Bloch bands $\left| \pm 2 \hbar k_{0}\right\rangle=\left|n_{b} \cdot 4 \hbar k_{0}-2 \hbar k_{0}\right\rangle \equiv\left|n_{b}\right\rangle$, while the bosonic-mode quadratures are encoded in the position $\hat{x}$ and quasimomentum $\hat{q}$ of the atoms. The qubit can be initialized in an arbitrary state by preparing the atoms in the corresponding position of the Bloch spectrum. This can be done by applying a Doppler-sensitive Bragg pulse [49]. Due to momentum conservation, the process entails a discrete momentum kick of $\pm 2 \hbar k_{0}$. By controlling the share of atoms that gain positive or negative momentum, as well as the relative phase between the Bragg pulses, the effective qubit can be initialized in any superposition of $\sigma_{x}$ eigenstates [as defined in Eq. (7)].

Both the momentum (and correspondingly the state of $\sigma_{x}$ ) and, in principle, the position can be measured with absorption imaging techniques [33]. For the former, standard time-offlight imaging can be used, as performed by simultaneously deactivating both the lattice beams and the dipole trapping potential and then detecting the atoms in the far field after a given free expansion time. While the reconstruction in this way is possible with a high precision [42], achieving the required spatial resolution for an in situ position detection of the oscillation is experimentally challenging.

For completeness, we mention that the qubit operator $\sigma_{z}$ can also be directly measured via adiabatic mapping [33,50], but only when the system state is close to the avoided crossing in the Bloch spectrum. By accelerating adiabatically the lattice from $q \sim 0$ to $q \sim 0.5 \hbar k_{0}$, Bloch waves are mapped onto the free-particle momentum states. Such a process corresponds to a rotation in the effective qubit Hilbert space. The required adiabatic acceleration of the mapping can be performed by means of a continuous frequency chirp applied to one of the lattice laser beams.
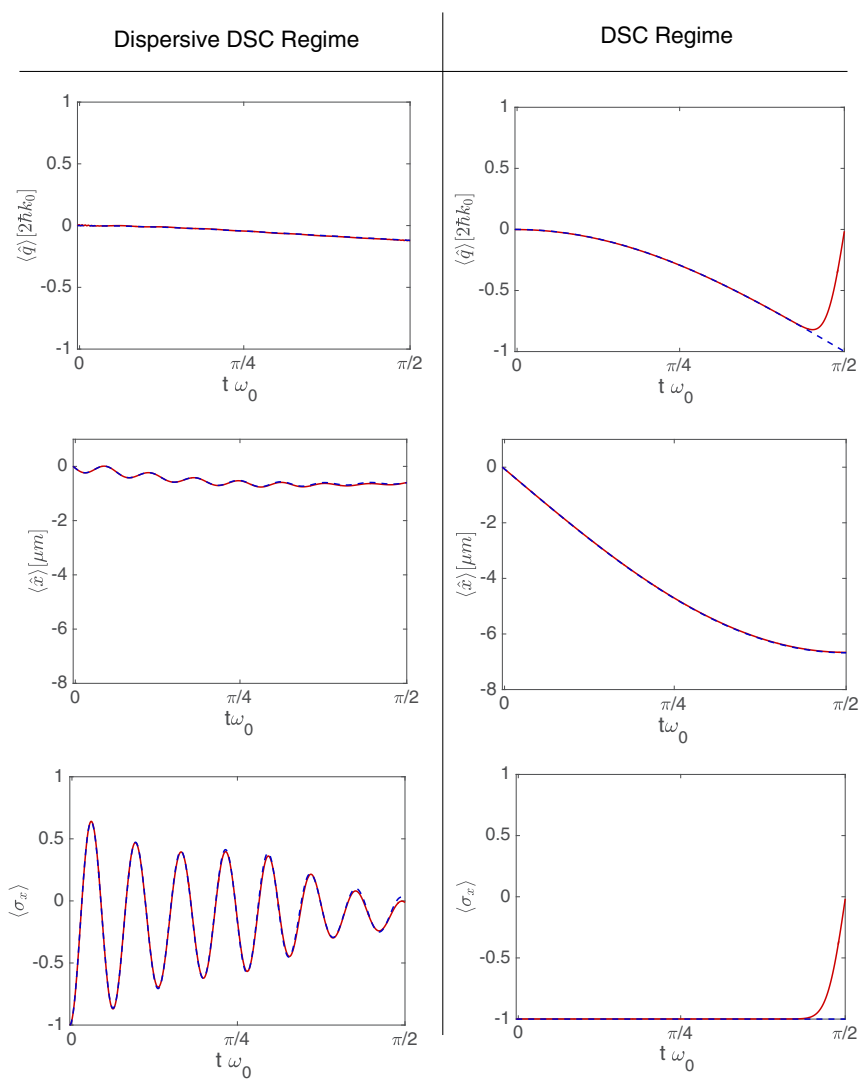

FIG. 2. Comparison between the full cold-atom Hamiltonian (red continuous line) and the quantum Rabi model (blue dashed line). The momentum is shown in units of $\hbar k_{0}$, while the position is shown in units of $1 / k_{0}$ and the coupling strength $g / \omega_{0}=5.18$. For the dDSC, the ratio of frequencies is given by $\omega_{q} / \omega_{0}=28.7$, while for the DSC regime, $\omega_{q} / \omega_{0}=0$.

\section{RESULTS}

Let us now consider the dynamics of the quantum Rabi model in the specific parameter regimes of interest for the proposed quantum simulation. Given that only very high values of the ratio between the coupling strength $g$ and the bosonic mode frequency $\omega_{0}$ are accessible, the rotating-wave approximation can never be applied and the model cannot be implemented in the Jaynes-Cummings limit. However, we will show that interesting dynamics at the crossover between the dDSC and the DSC regime can be observed for values of parameters that are unattainable with so far available natural implementations of the quantum Rabi model.

By means of numerical simulations, we have compared the dynamics of the full cold-atoms model in Eq. (1) with the corresponding effective quantum Rabi model in Eq. (6). Numerical simulations of the full model have been performed in the position basis, applying a discretization of the real space over more than $10^{3}$ lattice sites. The quantum Rabi model has been numerically simulated introducing a cutoff $(N>500)$ on the maximum number of bosonic excitations.

\section{A. DSC to dispersive-DSC regime transition}

In Fig. 2, we show the results of such numerical simulations, in different parameter regimes. The initial state $\left|\psi_{0}\right\rangle=$ 

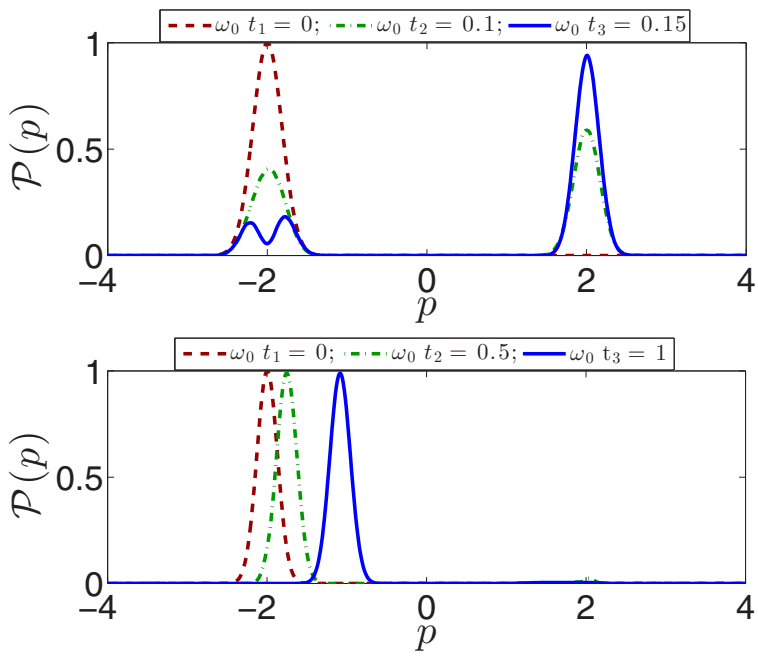

FIG. 3. Numerically evaluated real-momentum distribution of the cold-atom cloud during the dynamics shown in Fig. 2, at different evolution times. For the dDSC regime (upper panel), the Rabi parameters are given by $g / \omega_{0}=7.7$ and $g / \omega_{q}=0.43$. In this case, the initial wave function is transformed back and forth between two distributions centered on the states $\left|p= \pm 2 \hbar k_{0}\right\rangle$. For the DSC regime (lower panel), $g / \omega_{0}=10$ and $\omega_{0}=\omega_{q}$. In this case, the system is continuously displaced in momentum space until the maximum value of the momentum is reached.

$|q=0\rangle\left|n_{b}=1\right\rangle$ is given by the vacuum of the bosonic mode and eigenvectors of $\sigma_{x}$. Such a state can be obtained preparing the atomic cloud at the center of the harmonic trap and at the $q=0$ of the $n_{b}=1$ band of the Bloch spectrum, which corresponds to atoms prepared at $p=+2 \hbar k$. In all plots, the red continuous line shows the dynamics of the full model [cf. Eq. (1)], while the dashed blue line corresponds to the quantum Rabi model [cf. Eq. (6)]. The good agreement between the two simulations breaks down when the system state hits the border of the validity region of the quantum simulation. Different behaviors between the two regimes are more visible in the expected value of $\sigma_{x}$ which, in the DSC regime, is approximately a conserved quantity, as shown below.

Figure 3 shows the distribution $\mathcal{P}(p)=|\langle p \mid \psi(t)\rangle|^{2}$ of the atomic physical momentum $\hat{p}$, for different evolution times. The momentum distribution can be experimentally obtained via time-of-flight measurements, and gives a clear picture of the system dynamics during the quantum simulation of the quantum Rabi model. The cloud is initialized in the momentum eigenstate, $\left|p=-2 \hbar k_{0}\right\rangle=|q=0\rangle\left|n_{b}=0\right\rangle$. When the periodic lattice strength $V$ is large enough, the dynamics is dominated by the coupling between the $\left|p= \pm 2 \hbar k_{0}\right\rangle$ states. This case corresponds to the dDSC regime in Fig. 2. Otherwise, the dynamics is dominated by the harmonic potential, and the evolution resembles the quantum Rabi model in the DSC regime.

\section{B. Collapses and revivals}

We have first shown that our proposal is able to reproduce the dynamics of the quantum Rabi model at the crossover between the dDSC and DSC regimes. The analogy is broken when the value of the simulated momentum exceeds the
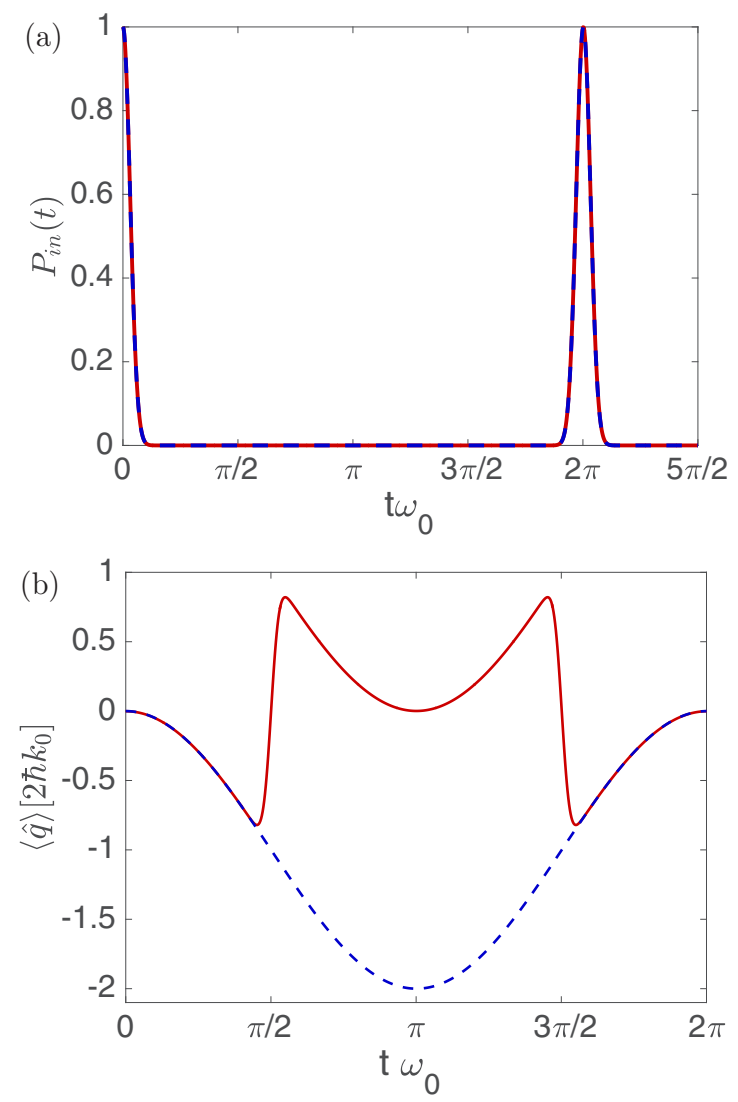

FIG. 4. Comparison between the full cold-atom Hamiltonian (red continuous line) and the QRM (blue dashed line). (a) The plot shows collapses and revivals of the initial-state population $P_{\text {in }}=$ $\left|\left\langle\psi_{\text {in }} \mid \psi(t)\right\rangle\right|^{2}$. The initial state is given by $\left|\psi_{\text {in }}\right\rangle=|q=0\rangle\left|n_{\mathrm{b}}=1\right\rangle$. The coupling strength is given by $g / \omega_{0}=5.18$, while the qubit energy spacing vanishes, $\omega_{q}=0$. In this limit, collapses and revivals correspond to harmonic oscillations of the atoms in the trap potential. (b) Temporal evolution of the quasimomentum in units of $2 \hbar k_{0}$, as in the right column of Fig. 2, for the long-time dynamics. Notice that the value of the quasimomentum is bound at $|q| \leqslant 2 \hbar k_{0}$ and that the different behavior between the Rabi and the periodic quantum Rabi model appears at the boundary of the Brillouin zone.

borders of the first Brillouin zone. When this is the case, the model represents a generalization of the quantum Rabi model in a periodic phase space. In the following, we show that collapses and revivals of the initial state, which represent the signature of the DSC regime of the quantum Rabi model, are matched by the full atomic model. Let us first review the quantum Rabi model dynamics, considering the initial state $\left|\psi_{\text {in }}\right\rangle=|q=0\rangle\left|n_{b}=0,1\right\rangle$. In the DSC regime, the system evolution is described by the approximated solution [43],

$$
|\psi(t)\rangle=e^{-i \hat{H}_{\mathrm{DSC}} t}\left|\psi_{\mathrm{in}}\right\rangle=-1 e^{-i \phi(t)} D\left[(-1)^{n_{b}} \beta(t)\right]|0\rangle\left|n_{b}\right\rangle,
$$

where $\phi(t)$ is a global phase independent of the qubit state, while $D[\beta(t)]$ is a displacement operator and $\beta(t)=$ $i \frac{g}{\omega_{0}}\left(e^{-i \omega_{0} t}-1\right)$. Accordingly, during the system time evolution, $\sigma_{z}$ is conserved, while the vacuum state is displaced into a coherent state that rotates in phase space and returns into the initial state with period $T=2 \pi / \omega_{0}$. This pattern of collapses 
and revivals is shown (blue dashed line) in Fig. 4(a) for the case in which the solution of Eq. (9) is exact $\left(\omega_{q}=0\right)$. The width of the peaks is given by the width of the momentum distribution of the initial state. As shown in Fig. 4(b), the periodicity of the momentum results in shifted values of $\hat{q}$ for the half period of the system dynamics. Notice that when the system is initialized in the state $\psi_{\text {in }}=\left(|q=0\rangle\left|n_{b}=1\right\rangle+|q=0\rangle\left|n_{b}=0\right\rangle\right) / \sqrt{2}$, the dynamics of Eq. (9) leads to Schrödinger cat generation, $\psi(t)=\left(|\beta(t)\rangle\left|n_{\mathrm{b}}=1\right\rangle+|-\beta(t)\rangle\left|n_{b}=0\right\rangle\right) / \sqrt{2}$. The size of the cat state is given by the maximum value of the displacement, $\beta_{\max }=2 \frac{g}{\omega_{0}}$, and so it is proportional to the coupling strength.

\section{CONCLUSIONS AND OUTLOOK}

We have developed a method to implement a quantum simulation of the quantum Rabi model for unprecedented values of the coupling strength, using a system of cold atoms in a periodic lattice. An effective two-level quantum system is simulated by the Bloch-bands occupation, while a single bosonic mode is implemented by the oscillations of the atoms in a harmonic optical trap. By means of numerical simulations, we have shown that highly nontrivial dynamics can be feasibly implemented within the validity region of the quantum simulation. In particular, we have shown that the crossover between the dispersive-DSC and the DSC regime could be experimentally analyzed. Furthermore, the proposed system represents a generalization of the QRM in a periodic phase space, which presents interesting patterns of collapses and revivals of the initial state in the DSC regime.

Notice that in the proposed quantum simulation, an ensemble of noninteracting systems evolves simultaneously. A natural extension of the present work is the inclusion of interatomic interactions, in order to implement a many-body system composed of interacting quantum Rabi models.

\section{ACKNOWLEDGMENTS}

We acknowledge useful discussions with Michele Modugno. This work was supported in part by the DFG (Grant No. We 5 1748-20). S.F. acknowledges funding from the PRESTIGE program, under the Marie Curie Actions-COFUND of the FP7 and from University Sorbonne Paris Cite EQDOL contract. E.R. and E.S. acknowledge funding from UPV/EHU Grant No. UFI 11/55, MINECO/FEDER Grant No. FIS201569983- P, and UPV/EHU Grant No. EHUA15/17. C.S. acknowledges funding from Fundación General CSIC (Programa ComFuturo), MINECO Project No. FIS2015-70856-P (cofinanced by FEDER funds), and CAM PRICYT Project No. QUITEMAD+ S2013/ICE-2801. Basque Government Grant No. IT986-16 Spanish MINECO/FEDER FIS2015-69983-P and FIS2015-70856-P.
[1] I. I. Rabi, Phys. Rev. 49, 324 (1936).

[2] E. T. Jaynes and F. W. Cummings, Proc. IEEE 51, 89 (1963).

[3] S. Haroche and J.-M. Raimond, Exploring the Quantum (Oxford University Press, New York, 2006).

[4] H. Häffner, C. F. Roos, and R. Blatt, Phys. Rep. 469, 155 (2008).

[5] A. Wallraff, D. I. Schuster, A. Blais, L. Frunzio, R. S. Huang, J. Majer, S. Kumar, S. M. Girvin, and R. J. Schoelkopf, Nature (London) 431, 162 (2004).

[6] J. Bourassa, J. M. Gambetta, A. A. Abdumalikov, Jr., O. Astafiev, Y. Nakamura, and A. Blais, Phys. Rev. A 80, 032109 (2009).

[7] T. Niemczyk, F. Deppe, H. Huebl, E. P. Menzel, F. Hocke, M. J. Schwarz, J. J. García-Ripoll, D. Zueco, T. Hümmer, E. Solano, A. Marx, and R. Gross, Nat. Phys. 6, 772 (2010).

[8] A. Fedorov, A. K. Feofanov, P. Macha, P. Forn-Díaz, C. J. P. M. Harmans, and J. E. Mooij, Phys. Rev. Lett. 105, 060503 (2010).

[9] P. Forn-Díaz, J. Lisenfeld, D. Marcos, J. J. García-Ripoll, E. Solano, C. J. P. M. Harmans, and J. E. Mooij, Phys. Rev. Lett. 105, 237001 (2010).

[10] A. A. Anappara, S. De Liberato, A. Tredicucci, C. Ciuti, G. Biasiol, L. Sorba, and F. Beltram, Phys. Rev. B 79, 201303(R) (2009).

[11] G. Günter, A. A. Anappara, J. Hees, A. Sell, G. Biasiol, L. Sorba, S. De Liberato, C. Ciuti, A. Tredicucci, A. Leitenstorfer, and R. Huber, Nature (London) 458, 178 (2009).

[12] C. Ciuti, G. Bastard, and I. Carusotto, Phys. Rev. B 72, 115303 (2005).

[13] C. Ciuti and I. Carusotto, Phys. Rev. A 74, 033811 (2006).

[14] S. Ashhab and Franco Nori, Phys. Rev. A 81, 042311 (2010).
[15] A. Ridolfo, M. Leib, S. Savasta, and M. J. Hartmann, Phys. Rev. Lett. 109, 193602 (2012).

[16] S. Felicetti, G. Romero, D. Rossini, R. Fazio, and E. Solano, Phys. Rev. A 89, 013853 (2014).

[17] R. Stassi, S. Savasta, L. Garziano, B. Spagnolo, and F. Nori, New J. Phys. 18, 123005 (2016).

[18] P. Nataf and C. Ciuti, Phys. Rev. Lett. 107, 190402 (2011).

[19] G. Romero, D. Ballester, Y. M. Wang, V. Scarani, and E. Solano, Phys. Rev. Lett. 108, 120501 (2012).

[20] T. H. Kyaw, S. Felicetti, G. Romero, E. Solano, and L. C. Kwek, Sci. Rep. 5, 8621 (2015).

[21] D. Z. Rossatto, S. Felicetti, H. Eneriz, E. Rico, M. Sanz, and E. Solano, Phys. Rev. B 93, 094514 (2016).

[22] D. Braak, Phys. Rev. Lett. 107, 100401 (2011).

[23] Q. T. Xie, S. Cui, J. P. Cao, L. Amico, and H. Fan, Phys. Rev. X 4, 021046 (2014).

[24] I. Travěnec, Phys. Rev. A 85, 043805 (2012); A. J. Maciejewski, M. Przybylska, and T. Stachowiak, ibid. 91, 037801 (2015); I. Travěnec, ibid. 91, 037802 (2015).

[25] V. V. Albert, G. D. Scholes, and P. Brumer, Phys. Rev. A 84, 042110 (2011).

[26] S. Felicetti, J. S. Pedernales, I. L. Egusquiza, G. Romero, L. Lamata, D. Braak, and E. Solano, Phys. Rev. A 92, 033817 (2015).

[27] D. Braak, J. Phys. B 46, 224007 (2013).

[28] K. Baumann, C. Guerlin, F. Brennecke, and T. Esslinger, Nature (London) 464, 1301 (2010). 
[29] D. Ballester, G. Romero, J. J. García-Ripoll, F. Deppe, and E. Solano, Phys. Rev. X 2, 021007 (2012).

[30] A. Mezzacapo, U. Las Heras, J. S. Pedernales, L. DiCarlo, E. Solano, and L. Lamata, Sci. Rep. 4, 7482 (2014).

[31] J. S. Pedernales, I. Lizuain, S. Felicetti, G. Romero, L. Lamata, and E. Solano, Sci. Rep. 5, 15472 (2015).

[32] J. Klinder, H. Keler, M. Wolke, L. Mathey, and A. Hemmerich, Proc. Natl. Acad. Sci. USA 112, 3290 (2015).

[33] I. Bloch, J. Dalibard, and W. Zwerger, Rev. Mod. Phys. 80, 885 (2008).

[34] E. Demler and F. Zhou, Phys. Rev. Lett. 88, 163001 (2002).

[35] K. Aikawa, A. Frisch, M. Mark, S. Baier, R. Grimm, and F. Ferlaino, Phys. Rev. Lett. 112, 010404 (2014).

[36] B. Lücke, J. Peise, G. Vitagliano, J. Arlt, L. Santos, G. Toth, and C. Klempt, Phys. Rev. Lett. 112, 155304 (2014).

[37] A. Recati, P. O. Fedichev, W. Zwerger, J. von Delft, and P. Zoller, Phys. Rev. Lett. 94, 040404 (2005).

[38] P. P. Orth, I. Stanic, and K. Le Hur, Phys. Rev. A 77, 051601(R) (2008).

[39] M. A. Cirone, G. De Chiara, G. M. Palma, and A. Recati, New J. Phys. 11, 103055 (2009).
[40] X. Lopez-Gonzalez, J. Sisti, G. Pettini, and M. Modugno, Phys. Rev. A 89, 033608 (2014).

[41] D. Witthaut, T. Salger, S. Kling, C. Grossert, and M. Weitz, Phys. Rev. A 84, 033601 (2011).

[42] T. Salger, C. Grossert, S. Kling, and M. Weitz, Phys. Rev. Lett. 107, 240401 (2011).

[43] J. Casanova, G. Romero, I. Lizuain, J. J. García-Ripoll, and E. Solano, Phys. Rev. Lett. 105, 263603 (2010).

[44] M.-J. Hwang, R. Puebla, and M. B. Plenio, Phys. Rev. Lett. 115, 180404 (2015).

[45] G. Ritt, C. Geckeler, T. Salger, G. Cennini, and M. Weitz, Phys. Rev. A 74, 063622 (2006).

[46] T. Salger, C. Geckeler, S. Kling, and M. Weitz, Science 326, 1241 (2009).

[47] E. K. Irish, Phys. Rev. Lett. 99, 173601 (2007).

[48] T. Salger, C. Geckeler, S. Kling, and M. Weitz, Phys. Rev. Lett. 99, 190405 (2007).

[49] C. Grossert, M. Leder, and M. Weitz, J. Mod. Opt. 63, 1805 (2016).

[50] A. Kastberg, W. D. Phillips, S. L. Rolston, R. J. C. Spreeuw, and P. S. Jessen, Phys. Rev. Lett. 74, 1542 (1995). 